\title{
An Investigation of Music Therapists' Experiences of Verbal Dialogue in Music Therapy Sessions
}

\author{
Siobhán Nelligan ${ }^{1 *}$, Tríona McCaffrey ${ }^{1}$ \\ 1 University of Limerick \\ *siobh.musictherapy@gmail.com
}

Received: 11 June 2019 Accepted: 7 February 2020 Published: 1 March 2020

Editor: Seung-A Kim Reviewers: Kaitlyn Kelly, Tim Honig

\begin{abstract}
This study presents a preliminary exploration of music therapists' first-hand experiences of engaging in verbal dialogue with clients in their clinical practice. To the authors' knowledge no previous studies have examined the role of verbal dialogue from the first-hand perspectives of experienced professionals working in the field. Three individual interviews were conducted with three accredited Irish music therapists. Four central themes emerged as a result of thematic content analysis: content and function of verbal dialogue, the use of verbal dialogue may contribute to professional ambiguity, returning to the music, and the dyadic relationship between musical and verbal exchange. The findings revealed verbal dialogue to be a topic of interest for the participants in this study, one which stimulated meaningful reflections about clinical practice. The implications for professional identity and clinical practice which arose distinguished verbal dialogue as a potential area for further research and professional discourse within the wider music therapy community. Suggestions were made for additional areas of learning that may assist in preparing trainee and newly-qualified music therapists for potentially challenging verbal encounters with clients.
\end{abstract}

Keywords: Key words: music therapy, verbal dialogue, verbal processing, counselling skills

\section{Introduction}

This paper presents an exploratory investigation of issues relating to music therapists' understandings of verbal dialogue and the perceived benefits and challenges of engaging in verbal dialogue with clients. Basic counselling or communication skills have an established place throughout many music therapy sessions, specifically to: open and close sessions, gather information and provide feedback about clients' musical experiences, establish trust, and to facilitate genuine encounters (Grocke \& Wigram, 2007; Lindblad, 2016). However, the applicability of verbal interaction remains a divisive topic within the literature (Aigen, 2014), with some authors arguing that the thera- 
peutic impact of musical engagement may be diminished if processed through higher cognition. Bruscia (2006) acknowledged that verbal dialogue may play a pertinent role with certain client groups but may be ineffective or inappropriate when working with others (nonverbal clients, for example). Certain theoretical approaches to music therapy place a greater emphasis on the use of verbal dialogue than others. For example, psychodynamic approaches have a strong verbal element (Priestley, 1994), while creative music therapy places greater emphasis on musical interaction (Nordoff, Robbins, \& Marcus, 2007). As a communicative medium, music has been described as "supra verbal" as it facilitates the expression of feelings and emotions that cannot be put into words (Beckwith, 1997; Grocke \& Wigram, 2007). Garred (2006) maintained that it is likely that the primary motivation for engaging with music therapy stems from the inherent qualities of music itself. This makes music therapy an accessible medium to clients for whom verbal therapies are unsuitable, be this through disability or other barriers to engagement.

A number of authors have outlined the importance of context and relationship within the music therapy process. For example, Garred (2006) explained that the music in music therapy always exists within a "situation" which involves a therapist and a client relating to one another. Thus, the function of music in this instance is never as an "object" but as an inter-relational process that can only be understood within the context of that "situation." Similarly, McCaffrey (2013) posited that clinical musical improvisation can be understood as a form of inter-subjective discourse which promotes a deeper understanding of self in relation to others. Depending on circumstances, this understanding may or may not be supported through verbal processing, described by Nolan (2005) as "the talking that facilitates the therapeutic process during, and in response to, music making or music listening" (p.18). Nolan (2005) outlined the application of verbal techniques in providing clarity about the musical experience, developing client awareness across a number of domains, and providing the therapist with a means of obtaining pertinent information about the client and how to proceed. In this regard, verbal processing has been described as a dynamic process that unfolds over the course of therapy and refers more specifically to the process of assisting clients to make sense of their musical experiences.

Lindblad (2016) recognised the "supra verbal" qualities of music while simultaneously acknowledging the importance of words for comprehending and clarifying the musical experience on a conscious level. In a qualitative study of three music therapy sessions, three primary functions of verbal dialogue were identified. These included: 1) establishing trust to bring about a genuine encounter, 2) deepening the experience of the present moment, and 3) clarifying the client's life story. The sessions were carried out with research volunteers who had agreed to participate in a single, one-time music therapy session for the purpose of the study. Lindblad noted that verbal dialogue formed a dominant part of each session, and that in one case the therapist might not have used music at all if she had not been taking part in a research study which required her to do so. This was supportive of Nolan (2005) who noted that while verbal dialogue is rarely discussed in detail in the literature, it is likely to be widely used in practice.

The dearth of literature and resources on the topic of verbalisation in music therapy has been highlighted by previous authors (Lindblad, 2016; Schwartz, 2019). Grocke and Wigram (2007) suggested that comprehensive training in counselling skills was not necessary for music therapists but stressed that some basic skills could equip practitioners when exploring and processing music experiences with their clients. Grocke and Wigram (2007) also identified verbal dialogue as an inherent and inevitable part of the therapeutic process stating that "therapists talk with their clients, if for no other reason than to greet them at the start of a session and to get a sense of how the therapy is progressing" (p. 26). They listed seven ways in which music therapists engage verbally with their clients, to: 
1. Discuss with clients how they are feeling on the day, and what has happened since the last music therapy session

2. Find a focus for the music therapy session

3. Understand responses to musical experience

4. Gather more information about the client's experience that enables us to "reenter into musical experience with new insight and more developed therapeutic alliance" (Nolan, 2005, p.18)

5. Give feedback to the client about our own experience (where appropriate)

6. Give interpretations of what the client is doing, or what is happening (where appropriate), and

7. Close a session

The skilled helper approach developed by Egan (1994) introduced the SOLER acronym which outlined a set of skills which enable helping professionals to engage empathetically with their clients. These include: $\mathrm{S}$ - sitting squarely, $\mathrm{O}$ - open attitude, $\mathrm{L}-$ leaning toward, E - eye contact, and R - relaxed posture. Egan also outlined six skills fundamental to effective verbal communication which include active listening, reflecting, paraphrasing, open questions, probes (to elicit more information), and focused questions. Using Egan's model as a guideline, Grocke and Wigram (2007) suggested a further four skills which are necessary for practicing music therapists which include: providing choice; being comfortable with silence; establishing, developing and maintaining a therapeutic presence; and, closing a session.

Gooding (2017) suggested that the microskills model, also referred to as microcounseling, may provide a suitable framework for the verbal processing skills required by music therapists due to its applicability within a wide range of theoretical frameworks and its grounding in ethical practice. Gooding contended that this model could be easily integrated into music therapy training programmes as it was developed to teach basic counselling skills in a short period of time. The microskills model can be adjusted to accommodate varied levels of experience as well as particular theoretical orientations and areas of speciality.

The most thorough account of the role and application of verbal skills in music therapy was recently published by Schwartz (2019). This book delineates various forms of "communication" inherent in music therapy practice while offering practical guidelines for determining when and how verbal skills should be employed during the music therapy process, with consideration to a range of situational and contextual factors. This resource has bridged a gap in the literature by providing a comprehensive reference point for aspiring and practicing music therapists. Nonetheless, a gap remains regarding the first-hand perspectives and experiences of professionals working in the field. The present research project sought to address this gap by interviewing qualified music therapists who regularly use verbal dialogue as part of their clinical practice. A variety of terms such as verbal processing (Nolan, 2005), verbal exchanges (Schwartz, 2019), and verbal dialogue (Lindblad, 2016) have been used in the existing literature to describe the types of verbal communication that can occur within music therapy practice. The term verbal dialogue was deemed most appropriate for the purpose of this study and will be applied herein, in order to investigate the breadth of spoken exchanges that occur between client and therapist throughout the course of a music therapy session.

\section{Setting the context}

As a music therapy student, the first author developed the initial idea for this project through conversations with other students on the topic of verbal processing. These conversations revealed a level of apprehension around handling verbal scenarios which appeared to warrant further enquiry. Further investigation revealed little available research on the use of verbal dialogue in music therapy, despite widespread acknowl- 
edgement by therapists of its application within the field. The researcher thus developed an interest in investigating the topic further from the perspective of experienced therapists. The research design and subsequent approach to analysis was established in collaboration with the researcher's supervisor (second author).

\section{Challenges to the research}

Prior to commencing this research study, two potential challenges were evident. The first was providing a comprehensive definition of verbal dialogue and identifying the particular verbal interactions that the researcher was interested in exploring. It became clear, however, that the scope of verbal exchanges within the music therapy context was broad. Therefore, it was decided that the term "verbal dialogue" would be applied as a general descriptor for the spoken exchanges that occur during music therapy sessions. This allowed the researcher to consider all use of language (deliberate or casual) to be of equal relevance.

The second challenge presented itself in addressing the breadth and scope of music therapy practice. It was imperative to acknowledge that verbal dialogue does not form an integral part of all music therapy practice, and so questions emerged regarding the relevance of this topic for the overall professional community. Many music therapists work with non-verbal populations, and others operate from professional orientations which promote music as the primary catalyst for therapeutic growth. Thus, there are music therapists for whom the use of verbal dialogue bears little significance due to the professional context in which they work. With that in mind, there was no intention within the present research project to infer that verbal dialogue is an essential element of music therapy practice, and varied approaches to music therapy practice are acknowledged by the authors. However, specific applications for verbal dialogue have been identified within the music therapy context (Grocke \& Wigram, 2007; Lindblad 2016; Nolan, 2005; Schwartz, 2019), and certain schools of thought embrace verbal dialogue as a central component of the therapeutic process (Priestley, 1994). An investigation of first-hand clinical experiences of professional music therapists was therefore deemed appropriate to establish a preliminary understanding of verbal dialogue within music therapy sessions.

\section{Methodology}

\section{Participants}

Ethical approval was granted by the Faculty of Arts, Humanities and Social Sciences at the University of Limerick. The researcher employed purposive sampling when identifying suitable candidates. Participants considered eligible for this study were 1) Irish music therapists with Master's level training and full accreditation with the Irish Association of Creative Arts Therapists (IACAT), 2) who regularly employed verbal techniques in their sessions with clients, and 3) were willing to share their professional experiences of engaging verbally with clients. The researcher contacted three Irish music therapists (one male and two females with 10 to 20 years post-qualification experience) all of whom agreed to partake in the study. Participants were provided with a letter of informed consent outlining details pertaining to the nature of the study, confidentiality, and their right to withdraw. All participants were trained to Master's level and had experienced working in adult mental health settings, however experience with other client populations varied, including but not limited to: special needs, dementia, neurological conditions, and childhood mental health.

\section{Procedure}

Prior to the interview, participants were asked to sign a consent form outlining the conditions of participation and informing them that the interview would be audio recorded. They were reminded of their right to withdraw from the study at any time 
during the interview, or up to one week after the interview had taken place. Interviews lasted between 25-35 minutes and were carried out at locations of mutual convenience for both the researcher and participants. One interview was conducted via Skype.

Interviews were semi-structured and commenced with the question "What do you understand by the term "verbal dialogue" or "verbal processing" in relation to your work as a music therapist?" Participants were then provided with a short description of what was intended by these terms in relation to the research to ensure clarification. To encourage some level of consistency in terms of information gathered across participants, the researcher followed a general line of inquiry guided by the following questions:

- In what way are communication/counselling skills relevant to your work?

- How have you acquired these skills?

- How much time is typically spent talking with clients during music therapy sessions?

- Can you recall any examples of topics that might arise during verbal discussions with your clients?

- Can you describe when and where you might use verbal dialogue in your work with clients?

- Have you encountered any challenges in using verbal dialogue/processing with clients in your work?

- Have you encountered any benefits of using verbal dialogue/processing with clients in your work?

- Do you have any further thoughts on the topic of verbal dialogue in music therapy practice?

Interviews were recorded on a Zoom $\mathrm{H} 1$ handheld recorder and were transcribed verbatim on a Microsoft Word document. Recorded data was stored securely on the researcher's personal password-protected laptop and deleted after transcription. Each participant was offered the opportunity to choose their own pseudonym to protect their identity and to allow them to track their participation in the research if they so wished.

\section{Data analysis}

The researcher employed thematic content analysis as the primary method of data analysis. The procedure described below followed the six-step guide to data analysis recommended by Braun and Clarke (2006):

1. Familiarising yourself with your data

The researcher listened to the recordings a number of times to familiarise herself with the data set before, during and after transcription. Throughout this process the researcher kept a reflexive journal to document her thoughts, feelings, and observations to assist with subsequent interpretation of the data set. During transcription the researcher made every effort to preserve the individual nuances of the participants' dialogue by applying appropriate punctuation.

2. Generating initial codes

Codes were defined by the researcher as ideas and concepts which appeared meaningful in the context of the research question and the participants' individual experiences. The researcher read through each transcription manually highlighting words, phrases and quotes which stood out to her while adding handwritten reflective notes in the margins. Once initial codes were extracted, the researcher scanned the transcriptions again to identify whether recurrent codes existed across individual questions. Reflexive journaling continued throughout this phase and prominent subjective observations were discussed by both authors during supervision. 
3. Searching for themes

Once preliminary codes were identified, they were transcribed to a Microsoft Word document. Supporting quotes were included in the document. The researcher then began to establish loose categories from the codes, gradually refining these categories until a smaller number of potential units of analysis (i.e. potential themes) began to emerge. The reflexive journal assisted the researcher to keep track of any subjective observations that were emerging and how these may have been influencing emerging themes at this stage in the process. This was further explored during the supervision process.

4. Reviewing themes

During this phase the researcher began to determine which units of analysis (i.e. themes) were most prominent and relevant to the research topic. At this point the contents of each category were revised, and some categories were made redundant. The researcher referred regularly to her reflexive journal throughout this process to minimise researcher bias. Categories were reviewed several times until the researcher was satisfied with the resultant themes. The supervision process aided objectivity during this process.

5. Defining and naming themes

During this phase the researcher began to develop detailed descriptions of each theme. Preliminary theme names were also revised and given more attention during the supervision process. Final theme names were chosen to serve one of two functions: 1) to provide a simple, descriptive account of a finding; or 2) to give voice to a recurrent phrase or concept used by participants.

6. Producing the report

Theme descriptions were finalised and the most appropriate quotes were extracted for presentation in the final write up. This phase involved a joint effort between both authors to ensure that a balanced perspective was maintained during the presentation of findings.

\section{Findings}

Individual interviews were conducted with three professional music therapists about their experiences of implementing verbal dialogue with clients as part of their clinical music therapy practice. Participants were assigned the pseudonyms Lily, Jane, and Brian. The following themes emerged during analysis across each of the interviews: content and function of verbal dialogue, the use of verbal dialogue may contribute to professional ambiguity, returning to the music, and the dyadic relationship between verbal and musical exchange.

\section{Content and function of verbal dialogue}

The content and function of verbal dialogue reflected specific accounts given by participants about the nature of the verbal exchanges they had experienced with clients. Participants identified two main functions of verbal dialogue in their practice: maintaining an authentic relationship and facilitating and processing musical experiences.

\section{Maintaining an authentic relationship}

All participants remarked that verbal dialogue assisted them in establishing meaningful connections with clients while normalising the concept of music therapy, particularly during the early stages of the therapeutic process. Jane highlighted how seemingly superficial verbal interactions may provide pertinent information about a client's well-being, noting that "even when someone walks into the room and they say "hello, how are you," and sit down, I'm thinking as a therapist ... so... I'm noticing how they present that "hello," you know, or their response to that." She returned to this later in the interview by saying: 
Jane: Yeah but it's interesting that chit-chat [] I guess the chit-chat doesn't have to be part of therapy. It's part of my therapy, and probably most people's, 'cause it's normal humanistic interaction.

Siobhán: Yeah, yeah. And I suppose it does vary very much amongst professionals...

Jane: Yes

Siobhán:.. and probably client groups as well ...

Jane: Yes, 'cause, like, with teenagers you might need to normalise it. I think it's about normalising it, isn't it? You know ... like, with the people with dementia, you know ... they're not used to coming into a room and being asked to play an instrument. And a lot [] often don't want to play the instruments, so you have to sit down and say, you know 'How are you getting on in hospital?', or some kind of opener ... is, you know ... it's normal”

Brian echoed these sentiments by asserting that "the session starts when you're walking down the corridor." He remarked on the habitual nature of verbal dialogue by stating, "It's a human interaction. We're both human at the end of the day."

\section{Facilitating and processing musical experiences}

Participants distinguished the facilitation or instructional part of the music therapy session from the processing piece that occurs after a musical exchange. In contrast to interactions that were considered part of everyday conversation, these interactions served a specific therapeutic function. Lily provided the most detailed example of this:

Lily: Emm... so I would say, after an improvisation you might do verbal reflection, or verbal processing... em ... all the verbal stuff that helps to sort of consolidate what's happened in the music, all the verbal stuff that helps clients to sort of ... understand, em ... what's happened, and garner some sort of insight from what's happened in the music... so that would be my sort of instinct. 'Cause I think... 'cause there's a lot of verbal work outside of that. There's quite a bit of verbal in the work I do... but the processing ... but when you say verbal processing what I really think of is that piece after a musical exchange.

Siobhán: And when you say that there are experiences outside of that, or that there are other verbal exchanges, what do you mean by that?

Lily: There's a lot of verbal... exchange, verbal dialogue, in terms of... making the abstract more concrete. The facilitation bit I'd say. So, there's a lot of verbal work that goes on, in mental health anyway, I always think there's a lot of... it's probably half and half almost in some of my groups, em, so all the facilitative stuff, say, and all the instructional stuff, em, all that I think is important as well.

In relation to the verbal content, which emerges during verbal processing, the topics of conversation identified by participants varied between everyday issues, musical background and interests, and disclosures about personal issues such as family, relationships, pain, loss, sexual experiences, and physical symptoms. In relation to mental health, Lily noted that recovery concepts such as hope, personal responsibility, personal resourcing, and personal identity were common topics of conversation. She also noted that clients often want to talk about past experiences. Brian noted that adult clients in mental health settings would frequently disclose personal information in music therapy that they did not want shared with other healthcare professionals by saying, "the male clients I see in mental health will tell me things they would never tell the nurses." Similarly, Jane noted that clients in mental health settings would sometimes use the music therapy space to speak about issues relating to drugs, medication, and doctors.

\section{The use of verbal dialogue may contribute to professional ambiguity}

The use of verbal dialogue engendered ambiguity amongst two of the three participants with regards to their professional identity. While reflecting on their experiences of professional practice, participants began to examine the extent to which verbal dialogue was appropriate within the music therapy session. Differences in opinion 
emerged regarding roles, responsibilities, and professional identity, and appeared to expose a complexity in defining the global perimeters of music therapy practice.

Jane expressed confidence in her role as a music therapist, affirming, "I suppose I'm very clear in that I'm a music therapist, not a counsellor, or a psychotherapist." She noted that the verbal techniques she cultivated during her early career were designed to assist people to "talk rather than trying to interpret." She articulated a concern that music therapists potentially move beyond their sphere of professional competence when they begin to delve into the verbal realm with clients by saying, "the other danger is [] that people think they can do more than they can." She recalled scenarios in which she had referred clients for psychotherapy once the emphasis of the sessions began to shift towards a greater need for verbal processing. In contrast, Brian identified strongly with his role as a "therapist" stressing that there should be no limits to the verbal material handled by music therapists, "Why should there be? And [] if we engage in our own therapy, and in life-long learning, we can build up those skills." He spoke with clarity throughout the interview about his professional responsibility to inquire after the well-being of his clients, many of whom he had worked with for several years. Part of this responsibility involved recognising when a client may benefit from additional professional input.

Lily concurred with Brian's sentiments that music therapists should be prepared to process any verbal material that emerges for a client, however she recounted feeling a deficiency in these skills early in her career:

And I think... that was the major sort of surprise for me, or shock even, for me, when I finished my training, and I started work... actually, particularly in mental health, em, and particularly with adults, I found that... I... I didn't feel skilled enough really, in the verbal, on the verbal side of things. I found that... I remember having that realisation, "Oh my God, people are gonna talk to me as well. So I felt... I felt pretty O.K. in terms of, em, the musical side, and really the ... the clinical side for using music, or the therapeutic side of using music, but all the bits around it, I... I ... I got quite a land if I'm honest, because I didn't feel that I had the necessary skills... because people want to talk and ... and often times it takes you a while to sort of gear them, or move them, or shift them into the music. In mental health certainly. So ... depending on where they're at in their own... in their own, sort of, recovery journey... but ... yeah. That was one of the major learning pieces for me. I didn't quite realise there was going to be so much talking.

While speaking articulately throughout her interview about the effectiveness of music as a therapeutic medium, Lily expressed the greatest sense of conflict amongst participants about the implications of using so much verbal dialogue on her sense of professional identity as a music therapist:

And that struggle between ... that identity as a music therapist... and wondering whether you need to be more of a psychotherapist. And wondering whether you need to shift it to the music, because that's where you need to be... but is that where the client wants to be? So [] that's a kind of a... not a constant challenge, but it's... it's present a lot of the time.

While noting that communication and counselling skills are hugely relevant to the work, she also described the negotiation of verbal material as "uncomfortable territory" for music therapists, implying that this sense of ambiguity may be pervasive within the professional community, "Are we psychotherapists? Are we music psychotherapists? I'm not sure we're sure." She expressed a particular interest in "the questions we ask, the phrases we use, the language we use," contending that how music therapists use their language is more important than the amount of verbal dialogue they engage in. All three participants credited supervision and continuous professional development (CPD) in refining their verbal skills during the early years of their career.

\section{Returning to the music}

The concept of returning to the music was common amongst participants as they discussed the benefits of music as a non-verbal therapeutic intervention when the verbal realm has become blocked or dysfunctional. This theme represents points in the inter- 
views where participants moved beyond trepidations about roles and responsibilities, regaining confidence in their therapeutic skills, and chosen therapeutic medium.

Jane noted that while verbal dialogue formed a large part of her work, her tendency is to steer her clients towards music where possible, as this is her area of competency. She noted, "there can come a point where ... unless I can bring it into something deeper with my verbal skills, I need to bring it somewhere deeper in the music." Lily described moving back towards music when things "get stuck verbally." She also described moving back to the music in situations where a client's speech or thought patterns may be disorganised, to avoid compounding anything maladaptive. Jane echoed this by stating, "That is the real benefit of music therapy, I think, you know, with clients who are thought disordered, you know, that they don't have to talk."

Lily declared that there can be "comfort... in the verbal exchange, because that's what we know," but referred to the importance of "knowing when you need to move it to the music, "cause that's what we're skilled in." She spoke a lot about music as a "felt" or "lived" experience that cannot always be processed on a verbal level:

Often clients would say that they [] work on a much deeper emotional level when they stay in the music. And when we move it sometimes into the verbal, and when it becomes quite cognitive, they lose a bit of that felt experience, of [] being in the feeling, or being in the experience, or being in the memory, or being in the ... being in that desire they have for themselves.

Lily continued:

Sometimes [] it serves the client better not to put things into words. Ever. The experience of music therapy sometimes should not be put into words. Or what goes on for them internally... it doesn't all have to be put into words. They've experienced something, something has shifted, something has changed.

Both Lily and Jane expressed a concern about spending too much time in the verbal space, stating that clients may be more suited to psychotherapy if they are engaging in too much verbal dialogue. Lily and Jane also referenced situations where verbal dialogue can hinder the therapeutic process by facilitating avoidance and diluting the musical experience. Lily shared her thoughts on why some clients might want to stay in the verbal realm:

Like... part of the reason people are referred to music therapy is because they're having difficulty with the verbal. So... we have to be really mindful of that as well... because sometimes they draw you in to that space because ... it's a resistance of sorts... because they really don't function there. So, if they bring you in with them, you're going to stop functioning together.

She added that "we're moving into the more subconscious area through music ... so if we're drawn out of that you have to ask 'why?', you know?" Jane recalled deliberately steering a session back towards the music with a client who "might have talked the whole session, and it was possibly a defence from getting stuck in." However, Brian noted that on some occasions "you have to stay in the space," as the verbal material brought forward by the client may be sensitive in nature. Regarding "returning to the music" he remarked, "you don't even have to bring it back, it comes back itself," noting that something would usually emerge in the verbal exchange that would facilitate this, by saying "there'll be a reference, there'll be a song, there'll be a reference.. there'll be something." He described music as his "co-therapist," stating "if I can't do the work, my co-therapist can."

\section{The dyadic relationship between verbal and musical exchange}

Though participants expressed some conflicting attitudes towards the overall role of verbal dialogue in music therapy practice, each of them acknowledged a prominent interplay between music and verbal dialogue. This theme appeared to emerge as a resolution to earlier conflicts about roles and responsibilities as participants gradual- 
ly identified the complimentary nature of both mediums. Brian commented, "I think they're a dyad. They live together," also stating that they "complement each other... they feed each other." He described how "sometimes the music leads into the verbal, and sometimes the verbal leads into the music." He also observed that verbal dialogue is an intrinsic component of particular music therapy methods, such as song writing, lyric analysis, and receptive music listening.

Similarly, Lily described how music can provide a platform for verbal processing, "sometimes the music piece, or the music experience piece in therapy [] allows [clients] to find the words," while Jane suggested that verbal processing can help to "illuminate what you're doing in the music." Lily further illustrated how this dyadic interplay can unfold:

we might be in a processing piece... but, em, you might find that we're getting a bit stuck $\ldots$ and I'd really just name that and move it back to the music... and then when we come back to the processing something has shifted, or there's something new ...

Further illustrating the complicated and interweaving relationship between music and verbal dialogue in music therapy practice, both Jane and Brian provided accounts of communicative exchanges with clients who have limited capacity for verbal interaction. Jane recalled interactions with clients with dementia commenting that "even though they're very confused, I think there's quite a richness in their verbal material as well as their musical [interaction]." Brian took this a step further by categorising an intense vocal exchange with a non-verbal client as "verbal dialogue," which involved "eye contact," "smiles," and "hand gestures." Brian justified this through use of the following example:

he wants to sing "Old MacDonald" all the time, and... what he does, he goes "Ee, aye, ee, aye, OH". And the "OH" goes on ... can go on for something like six minutes ... and I'm doing it at the same time, where we're both ... every now and then we both take a breath together (takes breath), and we go "OH ..."

There was an acknowledgement by all participants that the relationship between verbal dialogue and music is more prominent when working with certain client groups, particularly mental health populations, and that the preferred style of the therapist also comes into play.

\section{Discussion}

The present study sought to provide a preliminary inquiry into the experiences of Irish music therapists employing verbal dialogue within their music therapy practice. The self-reflective narratives that emerged in this study exposed verbal dialogue as a pertinent clinical issue, which participants were eager to explore in more detail. Four primary themes emerged, the content and function of verbal dialogue, the use of verbal dialogue may contribute to professional ambiguity, returning to the music, and the dyadic relationship between verbal and musical exchange. Although the use of verbal dialogue may contribute to professional ambiguity emerged as a compelling individual theme, issues relating to professionalism maintained such a strong presence throughout each interview that the researcher found it challenging to consider it in complete isolation from other themes. The authors hope that the present discussion will illustrate this undercurrent of professionalism with greater coherence. Many of the concerns that emerged appeared to reflect hesitations regarding participants' personal sense of professional identity, rather than revealing concerns about the professional community on a whole. However, issues surrounding professional identity have arisen previously within the Irish context (McCaffrey, 2013) and may warrant further attention. This may partly reflect the fact that, unlike their counterparts in the United Kingdom, Irish music therapists still await statutory recognition.

Within the theme of content and function of verbal dialogue the contribution that verbal dialogue makes in maintaining authentic relationships with clients was identified. 
Participants believed that casual verbal interactions were necessary to "normalise" the concept of music therapy. This finding is congruent with service user accounts of music therapy in adult mental health settings, where ambiguity surrounding the concept of music therapy has been identified as a potential challenge in the initial stages of therapy (McCaffrey, 2018). The process of "normalising" also appeared to serve an additional function of neutralising power dynamics within the relationship, with one participant, Brian, commenting on the human quality of these exchanges.

Additionally, participants made a clear distinction between instructional skills and processing skills, with the implication that verbal processing skills overlap on some level with psychotherapeutic skills. This reflected Nolan's (2005) description of the role of verbal processing in music therapy and Schwartz' (2019) distinction between various forms of verbalisation during the music therapy process (e.g. verbal framing, verbal exchanges, verbal interactions, and verbal interventions). They also reported encountering a range of verbal interactions in their sessions, from everyday exchanges to personal disclosures that held meaning for the client. It appeared, for the most part, that participants did not actively seek out sensitive information from their clients but that clients were forthcoming with personal issues due to the sense of trust and safety they experienced within the therapeutic environment. It could be argued that the emphasis on the therapeutic relationship, which is central to music therapy practice (Bruscia, 1998), may invite personal disclosures regardless of whether these are actively sought or encouraged by the music therapist. Therefore, music therapists may require training to prepare them for such verbal disclosures even if their preferred style of working does not place emphasis on verbal dialogue.

Surveys conducted with student music therapists have previously revealed verbal and communication skills to be a domain that requires greater attention on training programmes (Clements-Cortes, 2015). While not all music therapists may require the same level of competence in verbal techniques due to working with non-verbal populations, there may be a basic level of training that is appropriate for entry-level graduates to ensure that they are equipped to deal with diverse verbal scenarios. This corresponds with Grocke and Wigram's (2007) recommendations that comprehensive counselling skills are not essential to music therapy practice, but some training is evidently beneficial. Edwards and McFerran (2004) previously made recommendations about educating music therapy students about sensitive topics such as childhood sexual abuse, stating, "It is a challenging responsibility to ensure that students are prepared for the work that awaits them in the field, and in particular are alerted and appropriately prepared for the requirements of working therapeutically with vulnerable populations" ( $\mathrm{p}$. 335). The present authors concur that addressing potentially triggering topics within the safety of a training programme may buffer distress if these issues arise unexpectedly for students on clinical placement.

All participants expressed a confidence in music as their chosen therapeutic medium and spoke clearly about the benefits that music can bring about. However, two of the three participants expressed ambiguous feelings in relation to their perceived roles and responsibilities regarding the verbal material they encounter during their sessions. While all participants noted that maintaining a balance between verbal and musical interaction was their preferred style of working, specific concerns arose for Lily and Jane in relation to how much time should be spent in the "verbal space" and knowing how and when to direct clients back towards the music. Brian did not share in this uncertainty, expressing confidence in the capacity of music therapists to develop necessary verbal skills throughout their career. This pointed to an important contrast in perspective which mirrors long-standing debates over the role of verbal dialogue in music therapy (Aigen, 2014). It is possible that preferred approaches to practice and experiences of different working environments influenced participants' perceived sense of professional boundaries. While participants did not identify a need for limitations to be placed on the quantity of verbal material music therapists engage in, they emphasised the responsibility held by individual practitioners to seek additional training or support where necessary and to recognise when a referral to psychotherapy may 
be more appropriate. While differences in opinion emerged, participants were united in their concern for the welfare of their clients, expressing a strong commitment to their overall role as "therapist". Personal psychotherapy, clinical supervision, continuous professional development (CPD) and professional experience were credited as avenues for developing appropriate verbal skills, particularly within the early years of professional practice.

A prominent theme which emerged naturally across each interview was the concept of "returning to the music" when verbal dialogue was unsuitable or when the therapeutic process had become blocked. This theme reflected participants' strong belief in the benefits of their primary therapeutic medium and represented a stage in the interview process where participants began to reclaim a sense of trust in their professional ability. Embedded within the concept of "returning to the music" were deliberations regarding the ways in which verbal dialogue may hinder progress by facilitating avoidance or diluting the embodied experience of music-making. However, while Lily and Jane spoke clearly about taking deliberate actions to guide clients "back to the music", Brian asserted that the therapeutic process moves back toward the music naturally. His description of the music as his "co-therapist" revealed a nuanced perspective on how the client's process becomes guided in the direction of music. A larger data set may have permitted deeper inspection of these nuances, nonetheless, the concept of "returning to the music" was a common theme across participants.

Although conflicting thoughts regarding verbal dialogue emerged within, between, and throughout the interviews, as each interview unfolded it became clear that both music and verbal exchange were viewed as valuable tools with which to support the therapeutic process. Improvisational music was described as a "felt" or "lived" experience which may supersede verbal dialogue at times. Participants identified moments where music could be employed skilfully to move beyond "blocks" in cognition. It was equally recognised that verbal processing could assist clients to cognitively consolidate this "felt experience", presenting opportunities for insight to develop. The perceived role of the music therapist was to intuitively gauge whether the client's process was best supported through musical engagement or verbal reflection from moment to moment. Additionally, it was observed that verbal dialogue is embedded in particular music therapy methods such as song-writing and lyric analysis. Short (2013) previously outlined some of the unique verbal scenarios encountered by music therapists when navigating the provocative lyrical content that may be introduced by clients when exploring rap as their chosen musical genre. It is evident that the verbal scenarios encountered by music therapists may be unique in certain settings.

While the term "verbal dialogue" was intended to refer solely to spoken exchanges, it became apparent that participants' understanding of "dialogue" was not limited to verbal exchanges. The breadth of communicative exchange in music therapy has been described by Schwartz (2019) as comprising words, language and speech; musical interaction; and gestures, physical actions, affect and posture. Participants in this study illustrated how music therapists often facilitate meaningful communicative interactions with clients whose capacity for verbal dialogue is limited. Clinical examples provided by Jane and Brian demonstrated that in order to facilitate such exchanges music therapists employ an expansive communicative repertoire which amalgamates verbal dialogue, vocalisations, and other musical techniques. It is therefore likely that rather than viewing the musical and verbal realms as mutually exclusive, music therapists generally possess a broader definition of the concept of "dialogue" or "communication" than spoken language permits. This may provide a partial explanation for the professional uncertainties that arose for participants when considering the musical and verbal realms in isolation. Participants appeared to resolve these concerns by determining that music and verbal dialogue operate as a dyad within the music therapy context, each affording unique possibilities but ultimately enhancing the therapeutic potential of the other. The dynamic interplay between conscious awareness and embodied experience has previously been delineated in relation to the art therapy process by Blomdahl and colleagues (2018). Their description of the "inner dialogue" as a pri- 
vate, unfolding experience of self is echoed in the present finding that music and language exist within a "dyadic" relationship which supports the unfolding internal and external processes of the music therapy client.

In respect of the present findings, the authors wish to make some recommendations for the incorporation of verbal skills training on education programmes and for future research.

\section{Limitations}

An obvious limitation to the present study was the small participant pool. Some interesting contrasts in opinion emerged in the data set which may have been possible to explore further had the study included a larger data set. While the small scale of the study limits the generalisability of findings, the authors suggest that some of the emerging themes may offer a foundational reference point for future enquiry into this topic.

The present study was completed as part of an academic programme, thus placing time constraints on elements of the analysis process. As a result, sufficient time was not available to engage in a member-checking process. This limitation was buffered through ongoing consultations with the lead researcher's academic supervisor (the second author of this publication) and through maintaining an ongoing reflexive journal which was consulted regularly during the data analysis phase. However, it is acknowledged that member-checking would have minimised researcher bias and increased the validity of interpretations.

While conducting and analysing interviews, it occurred to the lead researcher that the term "verbal dialogue" may have been too limiting to the topic of the research. Much of what was discussed throughout the interviews could better be described as "interactions" or "exchanges", and the skills required for these forms of communication were not solely applicable to spoken language as the term "dialogue" might imply. The distinction between various types of verbalisations in music therapy has been delineated more recently by Schwartz (2019). Additionally, all three participants had experience working in mental health settings, where it was acknowledged that verbal skills are particularly relevant. This meant that each participant had experience in negotiating verbal scenarios, identifying verbal skills as an integral element of their work. While this provided rich information on the use and necessity of these skills within certain contexts, it did not provide space for an alternative view. While reference was made to verbal exchanges with people with dementia and special needs, participants emphasised that their experience in mental health was more relevant to the topic. In hindsight, it may have been possible to extract richer information on other populations had the researcher probed participants further.

\section{Recommendations}

Based on the present findings, the authors support recommendations for basic verbal skills training on music therapy programmes. Previous authors have outlined paradigms which may be suitable for inclusion without over-loading already burdened programmes (Gooding, 2017; Grocke \& Wigram, 2007; Schwartz, 2019). Training programmes may wish to focus on supporting trainees to facilitate the verbal elements of widely-used music therapy methods such as songwriting and lyric analysis and how to skilfully shift between the verbal and musical domains during pivotal moments in sessions. It is appreciated and advised that individual qualified practitioners must take responsibility for seeking additional training when pursuing specialised clinical domains. However, basic confidence in these core areas may contribute to overall professional competence upon entering the field.

In light of the present findings, the authors propose that education programmes provide some pre-emptive support to trainees regarding the array of verbal exchanges they could encounter in clinical practice. Future research may help to quantitatively establish specific areas of educational need, however initial recommendations relate to 
social issues identified during analysis including: family and relationship issues, grief and loss, trauma, personal identity, and gender and sexuality. Equipping trainees with a basic awareness of such issues may help to buffer feelings of overwhelm and enhance their capacity to respond appropriately in moments of uncertainty. Furthermore, while previous authors have begun to identify specific functions of verbal dialogue in music therapy (Grocke \& Wigram, 2007; Lindblad, 2016) additional research with clinical populations may generate new insights.

It is important to acknowledge that the participants chosen for this study were highly experienced in their work, and thus further research with student or newly-qualified music therapists may help to identify whether there are distinct verbal scenarios that music therapists find difficult to deal with in their early years of practice. Specific issues emerged in relation to participants' sense of professional identity. It may be worth exploring whether these issues are unique within the Irish music therapy context, or whether they are shared internationally. The authors also suggest that interdisciplinary research across the creative arts therapies may greatly enhance our understanding of this issue as the application of verbal skills is not isolated to music therapy practice.

\section{Conclusion}

The current study presented a preliminary exploration of the experiences of three music therapists on their use of verbal dialogue in music therapy practice. The interviews provided a rich data set which produced four central themes as a result of thematic analysis: content and function of verbal dialogue, the use of verbal dialogue may contribute to professional ambiguity, returning to the music, and the dyadic relationship between musical and verbal exchange. The topic of professionalism maintained a strong presence throughout each interview. Participants expressed concerns about ethical practice, professional responsibility, and remaining within their sphere of knowledge and expertise. Participants expressed a strong faith in music as their chosen therapeutic medium while acknowledging the interweaving nature of the verbal and musical dimensions of their practice. This study supports the findings of previous research by recommending the incorporation of basic counselling skills training on music therapy training programmes. The authors also propose that education surrounding social issues which impact upon clients may provide trainees with additional support and preparation for the types of verbal encounters that are likely to arise in clinical practice. Future research into student experiences of verbal dialogue may help to better inform specific areas of educational need.

\section{About the authors}

Siobhán Nelligan is a Music Therapist specialising with children and adolescents. She has a strong background in mental health, developmental disability and trauma-informed practice. She holds a BA (Hons) Psychology, MA Community Music, and an MA Music Therapy. Siobhán has extensive experience working with children and young people in a variety of contexts including community settings, primary and post-primary schools, mental health services, palliative care, and residential care settings. She currently works in a dual capacity as an Assistant Psychologist and Music Therapist with children and young people in care who have experienced complex developmental and relational trauma. Siobhán is experienced in the therapeutic application of creative and play-based mediums and is interested in collaborative, attachment-focussed and somatic approaches to therapy.

Tríona McCaffrey is a lecturer on the MA Music Therapy at the Irish World Academy of Music and Dance, University of Limerick, Ireland. She has a background in mental health practice and holds a BA (Music and Irish), MA and PhD (Music Therapy), and a Graduate Diploma in Psychology. Tríona is particularly interested in the role of music therapy in promoting mental health recovery and is co-founder of the Alliance for Recovery Research in Music Therapy, an international research group dedicated to active involvement of mental health service users in music therapy research. Her $\mathrm{PhD}$ focused 
on service user evaluation of music therapy in mental health, employing interpretative phenomenological analysis and arts-based methods to highlight service user's personal experiences. Tríona is also article editor for Voices: A World Forum for Music Therapy.

\section{References}

Aigen, K. (2014). The study of music therapy: Current issues and concepts. Routledge.

Beckwith, J. (1997). Music papers: Articles and talks by a Canadian composer (1961-1994). The Golden Dog Press.

Blomdahl, C., Wijk, H., Guregård, S., \& Rusner, M. (2018). Meeting oneself in inner dialogue: A manual-based phenomenological art therapy as experienced by patients diagnosed with moderate to severe depression. The Arts in Psychotherapy, 59, 17-24, https://doi.org/ 10.1016/j.aip.2017.08.006.

Braun, V., \& Clarke, V. (2006). Using thematic analysis in psychology. Qualitative Research in Psychology, 3(2), 77-101, https://doi.org/https://doi.org/10.1191/1478088706qp063oa.

Bruscia, K. E. (1998). Defining music therapy. Barcelona Publishers.

Bruscia, K. E. (2006). Case studies in music therapy. Barcelona Publishers.

Clements-Cortes, A. (2015). A survey study of pre-professionals' understanding of the Canadian music therapy internship. Journal of Music Therapy, 52, 221-257, https://doi.org/ 10.1093/jmt/thv006.

Edwards, J., \& McFerran, K. (2004). Educating music therapy students about working with clients who have been sexually abused. The Arts in Psychotherapy, 31, 335-348, https://doi.org/10.1016/j.aip.2004.06.006.

Egan, G. (1994). The skilled helper: A problem-management approach to helping (5th ed.). Brooks/ Cole.

Garred, R. (2006). Music as therapy: A dialogical perspective. Barcelona Publishers.

Gooding, L. F. (2017). Microskills training: A model for teaching verbal processing skills in music therapy. Voices: A World Forum for Music Therapy, 17(1), https://doi.org/10.15845/ voices.v17i1.894.

Grocke, D., \& Wigram, T. (2007). Receptive methods in music therapy: Techniques and clinical applications for music therapy clinicians, educators and students. Jessica Kingsley.

Irish Association for Creative Arts Therapists (IACAT). (2018). Code of conduct and ethics for creative arts therapists: Art therapists, dance movement therapists, drama therapists, and music therapists. www.iacat.ie/files/UserFiles/Code-of-Professional-Conduct-and-Ethics.pdf.

Lindblad, K. (2016). Verbal dialogue in music therapy: A hermeneutical analysis of three music therapy sessions. Voices: A World Forum for Music Therapy, 16(1), https://doi.org/ 10.15845/voices.v16i1.842.

McCaffrey, T. (2013). Music therapists' experience of self in clinical improvisation in music therapy: A phenomenological investigation. The Arts in Psychotherapy, 40(3), 306-311, https://doi.org/10.1016/j.aip.2013.05.018.

McCaffrey, T. (2018). Evaluating music therapy in adult mental health services: Tuning into service user perspectives. Nordic Journal of Music Therapy, 27(1), https://doi.org/10.1080/ 08098131.2017.1372510.

Nolan, P. (2005). Verbal processing within the music therapy relationship. Music Therapy Perspectives, 23(1), 18-28, https://doi.org/10.1093/mtp/23.1.18.

Nordoff, P., Robbins, C., \& Marcus, D. (2007). Creative music therapy: A guide to fostering clinical musicianship (2nd ed.). Gilsum, NH: Barcelona Publishers.

Priestley, M. (1994). Essays on analytic music therapy. Barcelona Publishers.

Schwartz, E. K. (2019). Basic verbal skills for music therapists. Barcelona Publishers.

Short, H. (2013). Say what you say (Eminem): Managing verbal boundaries when using rap in music therapy, a qualitative study. Voices: A World Forum for Music Therapy, 13(1), https://doi.org/10.15845/voices.v13i1.668. 